\title{
MS05-P01 | The Critical Role Of The fourth Position Of The Vxgfl Motif Of Pp2cs From Oryza Sativa In Regulation Of Aba Responsiveness
}

Han, Seungsu (Sungkyunkwan University, Suwon, KOR); Lee, Ji-Young (Duksung Women's University, Seoul, KOR); Lee, Yongmok (Sungkyunkwan University, Suwon, KOR); Kim, Tae-Houn (Duksung Women's University, Seoul, KOR); Lee, Sangho (Sungkyunkwan University, Suwon, KOR)

Regulation of abscisic acid (ABA) signaling is crucial in balancing responses to abiotic stresses and retaining growth in planta. An ABA receptor (PYL/RCAR) and a protein phosphatase (PP2C), a co-receptor, forms a complex upon binding to ABA. Previously we reported that the second and fourth positions in the VxGФL motif of PP2Cs from Oryza sativa are critical in the interaction of PP2Cs with PYL/RCARs. Considering substantial effects of the VxGФL motif on ABA signaling outputs, further comprehensive characterization of residues in the second and fourth positions are required. Here we surveyed the second and fourth positions of the VxG $\Phi \mathrm{L}$ motif by combination of biochemical, structural and physiological analyses. We found that the fourth position of the VxGФL motif, highly conserved to small hydrophobic residues, was a key determinant of the OsPP2C50:OsPYL/RCAR interactions across subfamilies. Large hydrophobic or any hydrophilic residues in the fourth position abrogated ABA responsiveness. Comparison of crystal structures of OsPP2C50 mutants, S265L/I267V ("LV"), 1267L ("SL") and I267W ("SW"), in complex with ABA and OsPYL/RCAR3, along with energy calculation of the complexes, revealed that a bulky hydrophobic residue in the fourth position of the VxGФL motif pushed away side chains of nearby residues, conferring side-chain rotameric energy stress. Hydrophilic residues in this position imposed solvation energy stress to the PP2C:PYL/RCAR complex. Germination and gene expression analyses corroborated that OsPP2C50 AS and AK mutants modulated ABA responsiveness in Arabidopsis. Our results suggest that $A B A$ responsiveness could be finely-controlled by the fourth position of the VxGФL motif on PP2Cs. 